\title{
General Operational Protocol for Coherence. Central Limit Theorem as Approximation
}

\author{
Maria K. Koleva \\ Institute of Catalysis, Bulgarian Academy of Sciences, Sofia, Bulgaria \\ Email: mkoleva_1113@yahoo.com
}

How to cite this paper: Koleva, M.K. (2021) General Operational Protocol for Coherence. Central Limit Theorem as Approximation. Journal of Modern Physics, 12, 605-622. https://doi.org/10.4236/jmp.2021.125039

Received: March 8, 2021

Accepted: April 13, 2021

Published: April 16, 2021

Copyright $\odot 2021$ by author(s) and Scientific Research Publishing Inc. This work is licensed under the Creative Commons Attribution International License (CC BY 4.0).

http://creativecommons.org/licenses/by/4.0/

\begin{abstract}
A general operational protocol which provides permanent macroscopic coherence of the response of any stable complex system put in an ever-changing environment is proposed. It turns out that the coherent response consists of two parts: 1) a specific discrete pattern, called by the author homeostatic one, whose characteristics are robust to the statistics of the environment; 2) the rest part of the response forms a stationary homogeneous process whose coarse-grained structure obeys universal distribution which turns out to be scale-invariant. It is demonstrated that, for relatively short time series, a measurement, viewed as a solitary operation of coarse-graining, superimposed on the universal distribution results in a rich variety of behaviors ranging from periodic-like to stochastic-like, to a sequences of irregular fractal-like objects and sequences of random-like events. The relevance of the Central Limit theorem applies to the latter case. Yet, its application is still an approximation which holds for relatively short time series and for specific low resolution of the measurement equipment. It is proven that the asymptotic behavior in each and every of the above cases is provided by the recently proven decomposition theorem.
\end{abstract}

\section{Keywords}

Decomposition Theorem, Central Limit Theorem, Notion of a General Operational Protocol, Notion of a Law, Coarse-Graining, Scale Invariance

\section{Introduction}

So far, the generally accepted view on the notion of coherence assumes that isolated systems synchronize the behavior of their constituents by means of establishing a steady interference pattern throughout the whole system by means of evening the frequencies, wave numbers and sustaining a constant phase shift of 
the waves emitted by the constituents. The quantum mechanical dualism wave-particle renders this mechanism to be proclaimed ubiquitous since atoms and molecules that constitute each and every object in the Universe are quantum objects.

However, a closer look on this consideration displays some fundamental flaws:

1) An interference pattern stays steady if only the environment is kept permanently the same. This precludes the application of the above idea to any system put in ever-changing environment which, however, is the vast amount of real systems. Thus, all living things, although constituted by atoms and molecules, exchange matter and energy with the environment (we eat, sweat etc.) which is ever-changing (day-night shift, seasonal changes etc.);

2) Interference patterns are unstable: they are vulnerable to tiniest local perturbations of the major characteristics which provide the interference: frequency, wave number and phase shift;

3) The interference pattern is static. The latter implies that once established, a pattern stays the same forever. Thus, the idea about coherence through interference becomes inapplicable to living organisms and open systems which change their current status in the process of exchanging matter/energy with a an ever-changing environment in a dynamical way;

4) The interference is an addition of waves and thus cannot launch chemical transformations.

The above considerations are persuasive enough to call for a new general idea about coherence which would be available for all complex systems put in an ever-changing environment.

To remind, the complex system is a new field of science that aims to provide answers to the questions: how parts of a system give rise to a variety of its collective behaviors, and how the system interacts with its environment. It is easy to enumerate examples of complex systems. These are, for example, the social systems whose constructive elements are the people; the brain as a biological system is composed out of neurons; molecules are formed out of atoms; the weather is formed out of air flaws. This new field of study of complex systems cuts across all traditional disciplines of science, as well as engineering, management, and medicine.

The intensive empirical examination that was going on in the last decades displays the remarkable enigma of their behavior: the highly specific for each complex system properties persistently coexist with certain universal, shared by each of them ones. Thus on the one hand, they all share the same characteristics, such as power law distribution and sensitivity to environmental variations, for example; one the other hand, each system has its unique "face", i.e. one can distinguish between an earthquake and heartbeat of a mammal. What makes the study of this coexistence so important is the enormous diversity of systems where it has been established. In order to get an idea about this vast ubiquity, let us present a brief list of such phenomena: earthquakes, traffic noise, heartbeat of 
mammals, public opinion, currency exchange rate, electrical current, chemical reactions, weather, ant colonies, DNA sequences, telecommunications, etc.

It is obvious that the coexistence of universal characteristics along with specific, even unique ones, suggests that its successful explanation must be insensitive both to the dynamical details of each and every system and to the statistics of the environmental impacts. Thus, the mathematical frame of any such explanation is rather closer to a general protocol then to a law in its traditional meaning. To remind, the notion of a law constitutes specific recursive relations between specific variables characterizing any given phenomenon which remain invariant on repetition. What is tacitly presupposed is that, on repetition, the environment must re-occur the same.

A general operational protocol which successfully explains the enigmatic coexistence of specific and universal properties shared by all stable complex systems has been put forward by the author in her book [1]. It consists of idea that this bizarre coexistence can be explained in the setting of a general operational protocol, called by the author boundedness, which asserts that a system stays stable if and only if the rates and amplitudes of both local and global exchange of matter/energy are permanently sustained not to exceed specific margins. It is obvious that this idea brings about a coherence of the response which comes from different parts of a system: indeed, the boundedness of rates and amplitudes implies establishing of long-range spatio-temporal correlations among distant parts of a system. In turn, a macroscopic coherence of any response of any complex system is established. The major advantage of the concept of boundedness consists of its central result which proves that the coherent response of any stable complex system comprises both a specific and a universal part: the specific one consists of a steady specific to a system pattern whose characteristics are robust to the details of the environmental impact and to the specific dynamics of the corresponding system, and the rest part which obeys universal distribution on a coarse-grained scale which is also insensitive to the details of the environmental impact and to the specific dynamics of the corresponding system.

Yet, 4 questions arise:

1) Which are the major characteristics of the macroscopic coherence? do they provide coexistence of universal and specific properties? Is the macroscopic coherence stable in a long run?;

2) Since this coherence is spontaneous, what is the general physical protocol which provides it so that to be universally available on the one hand, and robust to the details of any concrete dynamics on the other hand;

3) How the spontaneous development of internal fluctuations affects the macroscopic coherence? The importance of this matter lies in claim of the fluctuation-dissipation theorem which states that any response of any system exactly matches the behavior of an appropriate fluctuation. Then, why giant internal fluctuations do not destroy the permanent macroscopic coherence of the response; 
4) How the coherence interferes with the measurement.

In the setting of boundedness the answers above 4 questions turn intertwined. The general physical protocol governing the dynamics of the spontaneously executed physico-chemical processes has been put forward in [1] and consists in the following: local exceeds of energy are dissipated as emission of local acoustic phonons at a given spatio-temporal point and their absorption at another spatio-temporal point; local exceeds of matter are dissipated by means of targeted transportation of a specific constituents from a specific initial spatio-temporal point to a specific distant spatio-temporal point. Thus, the travel of matter waves between specific distant points serves as a general protocol for launching specific chemical reactions so that the input reagents and the output products are produced at distant points leaving at the same time the chemical reactions to be local events. Then, the global coherence appears as sequences of local emissions and absorptions of acoustic phonons and mater waves. The ubiquity of that mechanism is grounded on the ubiquitous presence of acoustic phonons and on the recently proved ubiquity of the matter wave emission [1] [2] in any system regardless to its nature and its physico-chemical characteristics.

The sequences of local acoustic phonon and /or matter wave emissions and absorptions are described mathematically through the properties of specially introduced for this purpose mathematical objects called by the author bounded irregular sequences (BIS) so that each term of any BIS consists of a unique piece of wave (local acoustic phonon and/or local matter wave). A systematic study of their behavior is provided in Chapter 1 and Chapter 2 of [1] where it is proven that they exhibit certain exclusive properties which are not shared by their unbounded and/or periodic counterparts. Some of these excusive properties come next.

The greatest importance of the proposed physical protocol is best pronounced through the proof that the power spectrum of any long-running BIS comprises additively two parts: a specific discrete pattern, called a homeostatic one, and a continuous band of universal shape $1 / f^{\alpha(f)}$ so that both the homeostatic pattern and the shape of the continuous band are robust to the details of the statistics of the succession of the members in any BIS. This result constitutes the notion of the called by the author decomposition theorem. Thus, the decomposition theorem proves that the response of any stable complex system is permanently macroscopic coherent and it consists of a specific steady pattern (the current homeostatic pattern) and the deviations from it which are also macroscopically coherent. Further, it is proven that the deviations from a homeostatic pattern obey a universal distribution whenever the continuous band in the power spectrum is a smooth one and does not signals out any special component. Thus, the central result of the decomposition theorem proves in most general terms the ubiquity of the coexistence of specific and universal properties for each and every complex system put in an ever-changing environment.

Next in the line of questions comes the following one: Under what conditions 
a homeostatic pattern remains stable and intact in a long-run? The proof of the decomposition theorem states that formally this is a condition about a uniform distribution of the zeroes of a BIS. Yet, the greatest advantage of the decomposition theorem is that the same condition provides not only a stable in a long-run existence of a specific coherent pattern (that is a homeostatic pattern) but a long-term stable functioning of the entire system by means of providing a BIS to form a stationary homogeneous process. In turn, the obtained robustness of the structure of a homeostatic pattern to the statistical and dynamical details of the interaction of the corresponding system with an ever-changing environment allows unambiguous separation of an object from its environment. Indeed, the notion of an object consists of its homeostatic pattern because the characteristics of a homeostatic pattern remain intact in an ever-changing environment. Moreover, one can define them regardless to the details of that environment.

Next in the present paper special attention to the role of the condition about uniform distribution of the zeroes for establishing a scale-invariant coarse-grained general distribution of any stationary BIS regardless to the details of the member succession in the original BIS, is paid. The importance of this attention lies in the considered in the section 3 highly non-trivial relation between the scale-invariance of the coarse-graining which produces a universal distribution of stationary BIS and the specific resolution of any measurement. Indeed, it is demonstrated that, although the notions of coarse-graining and resolution imply the same, namely non-discernibility of certain fine details, the superimposing of the resolution of any measurement, viewed as solitary operation of coarse-graining, onto scaleinvariant universal distribution, results in a rich variety of behaviors. Some of those cases are considered in section 3. It turns out that the relevance of the Central Limit Theorem appears as an approximation appropriate for specific relations between the characteristics of a given measurement and the characteristics of the corresponding universal behavior. Yet, the major result is that the asymptotic behavior of each and every stable complex system is governed by the decomposition theorem.

Outlining, the rich variety of behaviors is a highly non-trivial result of the impact of a measurement which, though being a specific form of coarse-graining, is not scale-invariant. It is worth noting once again that the universal behavior of a coarse-grained stationary BIS is scale-invariant unlike a measurement which is a solitary non-scale-invariant operation superimposed on the scale-invariant universal behavior.

The central for the present paper assertion states that the Central Limit Theorem (CLT) appears as a specific outcome of the role of measurement and turns out to be a good approximation to the decomposition theorem for relatively short time series. That is why, now I present the major clues and consequences of the decomposition theorem so that to reveal in the clearest way why and how CLT appears as an approximation to the decomposition theorem.

The high non-triviality of the above matter is best illustrated by the following 
consideration: the additive decomposition of a power spectrum to a homeostatic pattern and a continuous band does not involve any information about the details of the statistics and dynamics of the variations in any time series. To compare, the shape of the power spectrum of any unbounded time series explicitly depends on concrete statistics of the time series. The robustness of any homeostatic pattern and the robustness of the shape of the corresponding continuous band render the resultant decomposition generic property of each and every stable complex system. Thus, the decomposition theorem appears as the widest grounds for the concept of boundedness and thus it serves for the widest grounds for the description of the behavior of all stable complex systems.

It is worth noting that the decomposition theorem is fundamentally different from the Central Limit Theorem which serves as the widest grounds for the traditional theory of probabilities. Indeed, while the Central Limit Theorem holds for independent random variables (yet unbounded), the decomposition theorem holds for arbitrary variables provided the latter are bounded (yet not independent). Thus, the subjects of both theorems have no common background. So, it is to be expected that such fundamental difference would have far going consequences one of which is subject of the present paper.

\section{Decomposition Theorem Revisited}

The major goal of the present section is to elucidate that the conditions which provide stable long-run functioning of a complex system ensure also a permanent robustness of a specific macroscopic coherent pattern (that is a homeostatic pattern) to the details of the interaction of the corresponding system with a non-specified ever-changing environment. It should be stressed that the persistent presence of accompanied continuous band in the power spectrum implies that not only the homeostatic pattern is coherent but the entire current response to any environment (non-homogeneous environment included) is permanently coherent. Thus, although the physical interactions are short-ranged, the boundedness of rates and amplitudes of exchanging matter/energy/information viewed as a general operational protocol, are sufficient to substantiate coherence of the response throughout the entire system.

The conditions which provide permanent long-term stable functioning of any complex system are: 1) permanent avoidance of resonances viewed as a general condition for permanent maintenance of the boundedness; 2) scale invariance of the uniform distribution of the zeroes of a BIS viewed as a condition for permanent maintenance of the smoothness of the shape of a continuous band. In turn the latter sustains the avoidance of resonances on each and every scale. It is proven also that at the same time, the scale invariant uniform distribution of zeroes provides universality of the distribution of a coarse-grained BIS.

The smoothness of the shape of the continuous band is considered in the first sub-section while the universality of the distribution is considered in the second subsection. 
Next the role of the condition about avoidance of resonances for permanent maintenance of the boundedness of amplitude is considered.

The proof of the decomposition theorem is rather lengthy and is a result of a highly non-trivial interplay of the above assumptions. Details are presented in the Appendix to Chapter 1, Chapter 2 and Chapter 10 of the [1]. Now I present the major clues which yield that theorem. The foremost clue is that the separation of the power spectrum to a specific pattern and a continuous band must stay steady regardless to the details of the succession of terms in the corresponding BIS. The latter implies that both the intensity of each and every component along with the structure of the discrete pattern must stay intact. The condition for keeping the intensity of those lines intact is permanent avoidance of resonances among their members. The sources for a resonance are two: 1) resonances which come from the interference between the discrete and the continuous band; 2) non-smoothness of the shape of the continuous band. Thus, in order to avoid the resonances among the lines which belong to a discrete pattern and those ones which belong to the corresponding continuous band, it is sufficient to impose additivity of their coexistence in a power spectrum. In Chapter 2 of [1] it is proven that the additivity is an exclusive property of the concept of boundedness. As a consequence of that additivity, the additive separation of the zeroes of BIS'es to those which belong to a discrete band and those which belong to the corresponding continuous band commences.

In the sub-section 2.1 the condition about scale-invariance of the uniform distribution of zeroes for providing the smoothness of the continuous part is considered. In the sub-section 2.2 the same condition is considered with regards to its role for providing a universal distribution of the coarse-grained structure of a BIS.

It should be stressed that each and every stationary BIS is a complex interplay between two types of stationary BIS: one that commences from the discrete pattern in a power spectrum and one that commences from the continuous band. Their properties are additively separated in the corresponding power spectrum but in the time series they are intertwined. A self-consistent procedure for their separation in a time series itself will be presented in the next section. Now the assumption that such separation is available is taken for granted. Next in this section only properties of BIS that come from the continuous part of a power spectrum are discussed. For the sake of brevity, next in this section I call a stationary BIS any BIS which comes from a continuous band.

\subsection{Universality of the Shape of the Continuous Band in a Power Spectrum}

Next the highly non-trivial role of the condition about the uniform distribution of zeroes of a stationary BIS for providing the universality and smoothness of the shape of the continuous band in a power spectrum is considered. In consequence, the latter occurs to be sufficient condition for substantiation of permanent additive separation of the homeostatic pattern and the continuous band in 
a power spectrum. Recalling that the latter additivity along with the considered next robustness of the shape of continuous band to the statistics of the corresponding time series are the key factors providing stationarity of the corresponding BIS, the condition about uniform distribution of the zeroes on each and every scale alone turns necessary and sufficient for providing stationarity and homogeneity of the corresponding process. And vice versa: at the end of the sub-section 2.2. it is demonstrated that the boundedness renders an exclusive property of thus formed stationary and homogeneous process to be a permanent sustaining of the uniform distribution of zeroes.

Let us now consider the non-trivial role of the condition about uniform distribution of zeroes at each and every scale for providing the smoothness of the shape of the continuous band in a power spectrum of a stationary BIS of length $T$. Given the condition $z T \rightarrow$ const to hold at every point of a BIS where $z$ is the corresponding zero in the window of length $T$. If a BIS has this property, its Fourier transform is given by:

$$
g(T f)=\lim _{\substack{T \rightarrow \infty \\ z T \rightarrow \text { const }}} \frac{1}{\sqrt{T}} \int_{0}^{T} \exp (i f x) f(x) \mathrm{d} x
$$

where $f$ is the frequency and $x$ is the current variable, e.g. the time.

The goal is to find $g(T f)$. It is majorized by:

$$
G(T f)=\lim _{\substack{T \rightarrow \infty \\ z T \rightarrow \text { const }}} \frac{1}{\sqrt{T}} \int_{1 / T}^{T} \exp (i f x) \mathrm{d} x=\lim _{\substack{T \rightarrow \infty \\ z T \rightarrow \text { const }}}=\frac{\text { const }}{f^{\frac{\alpha(f, T)}{2}}}
$$

Here the term $\frac{1}{f^{\frac{\alpha(f, T)}{2}}}$ is derived by the condition of continuation: indeed for any frequency different from $1 / T$, the corresponding component in the power spectrum fits the shape $\frac{1}{f^{\frac{\alpha(f, T)}{2}}}$ where $\alpha(f, T)>1$. Then, the continuity imposes the same shape to hold for the frequency $1 / T$. It is worth noting that the condition of continuation is a mathematical expression of the condition that all components in a continuous band participate equally in it and thus the continuous band does not signal out any special component. The question is whether the boundedness alone is sufficient for a unique determination of $\alpha(f, T)$.

It should be stressed that the condition about continuity of the power spectrum is verifiable only analytically since the shape $1 / T$ holds only at a point but in any of its infinitesimally small neighborhood the shape is $\frac{1}{f^{\frac{\alpha(f, T)}{2}}}$; on the other hand the computations of all sorts operate only with finite intervals and thus "smear out" the special role of a single point although not eliminating its special role.

Thus, the condition about continuity indeed turns necessary and sufficient to provide uniform contribution of all frequencies in the power spectrum. It is worth noting on the condition about the continuity is an immediate conse- 
quence of the condition about the uniform distribution of zeroes. Indeed, if otherwise, a special point would appear immediately.

It is worth noting also on inappropriateness of all types of "cut-off" technics for elimination of the considered here divergences because, although they succeed in elimination, neither of them is able to provide uniform contribution of all points. That is why the relevance of those technics is limited the field of critical phenomena only where the existence of a special point is an experimental fact, e.g. at phase transitions. On the contrary, the behavior of complex systems exhibit stable in a long-run behavioral pattern which does not signal out any special spatio-temporal point and thus the leading property of the corresponding behavioral pattern is its time-translational invariance.

It is worth noting that, if the condition for boundedness of amplitudes and rates does not exist, the Fourier spectrum would be white noise. Further, if the function is bounded but the condition about uniform distribution of zeroes is not imposed, $G(f) \sim \frac{1}{f}$.

In order to reveal better the highly non-trivial role of persistence of the condition $z T \rightarrow$ const over taking the limit $\rightarrow \propto$, the following paradox is presented. Given a BIS but the limit $T \rightarrow \propto$ is taken first. Then, taking into account the above result about the shape of $G(f) \sim \frac{1}{f}$, a logarithmic divergence of the variance is yielded. Indeed, according to its definition the variance can be presented as an integral over the entire power spectrum:

$$
\operatorname{Var} \propto \int_{1 / T}^{\infty} \frac{\mathrm{d} f}{f} \propto \ln \frac{1}{T}
$$

Thus, whenever the condition $z T \rightarrow$ const does not persist prior to the limit $T \rightarrow \propto$, the variance in Equation (3) diverges logarithmically on increasing the length of the time window $T$. It is worth noting that in this case the divergence holds regardless to what the value of the variance calculated by traditional means is. To remind the variance of any BIS, according to the traditional definition of variance, yields always a well-defined finite value and thus never diverges.

Moreover, the paradox escalates further through the following considerations. Taking into account that the variance of the fluctuations is a measure how probable the largest fluctuations are, the logarithmic divergence implies that on increasing the length of the time window, the largest fluctuations become more and more probable. In turn, this implies that the corresponding system is not stable, and its current state explicitly depends on the beginning and the end of the time window. Thus each system would appear as unstable and plastic so that any measurement induces changes in it regardless to how mild it could be. Further the paradox becomes even greater since these changes should appear in a universal way. The latter is a set by the robustness of shape of the continuous band to the nature of the system, to its dynamics and to the statistics of the corresponding time series. Alongside, it turns out that the moment of development 
of those fluctuations is not well-defined because it depends explicitly on the length of the time window and changes with it. In a nutshell the above paradox constitutes the conflict of $1 / f$ shape with time translational invariance. To remind, time translational invariance implies that a given phenomenon is reproducible if and only if there exists measurement such that the corresponding system neither selects nor signals out any special time or space point. Thus, the shape $1 / f$ is in conflict with time translational invariance.

Let us now consider the impact of the imposing the condition about the uniform distribution of the zeroes. The grounding fact is that in the frame of boundedness the variance of fluctuations in each and every time series is bounded and independent from the length of the time series. Thus, it is not divergent. But then how to overcome the difficulty with the logarithmic divergence which appears when the variance is presented through the integrated power spectrum? In order to overcome this difficulty I assume that the shape of the power spectrum follows the shape of $\alpha(f, T)$ established in Equation (2):

$$
1 / f \text { but } 1 / f^{\alpha(f, T)}
$$

where $\alpha(f, T)=1$ at $f=\frac{1}{T}$ and $\alpha(f, T)$ is a continuous monotonically increasing function of the frequency. The idea about the replacement of the shape $1 / f$ with $1 / f^{\alpha(f, T)}$ is an immediate outcome of the persistent priority of the condition $z T \sim$ const over the limit $T \rightarrow \propto$ presented in Equation (2).

The proof how the logarithmic divergence is eliminated is rather lengthy and highly non-trivial. I strongly recommend its careful study. It can be found in the Appendix to Chapter 1 and in Chapter 2 in the [1].

In order to get the idea how boundedness takes part in the proof let me present the following brief sketch:

Given a bounded time series and it is assumed that its power spectrum fits the shape $1 / f^{\alpha(f, T)}$. The question is whether the function $\alpha(f, T)$ can be determined by the boundedness alone. This question is highly non-trivial since it is well known that the shape of the power spectrum of any unbounded time series explicitly depends on its statistics.

The answer is positive and comes as follow: the boundedness implies that the amplitude of the fluctuations in any time series is independent from the length of the window where the measurement is made. That is:

$$
I_{\max } \propto T^{0}
$$

On the other hand, the inverse Fourier transform gives the following estimation of the amplitude of members of a BIS:

$$
I_{\max } \propto \sqrt{T} \int_{1 / T}^{\infty} \frac{1}{f^{\frac{\alpha(f, T)}{2}}} \mathrm{~d} f=\sqrt{T}\left(\frac{1}{T}\right)^{1-\frac{\alpha\left(\frac{1}{T}, T\right)}{2}}
$$

Equality of $I_{\max }$ from both presentations in Equations (5)-(6) sets the value 
of $\alpha\left(\frac{1}{T}, 1\right)=1$ and requires $\alpha(f, T)$ to be monotonically increasing function.

Further, it turns out that the time translational invariance sets $\alpha(f, T)$ to be a linear function. Indeed, the time-translational invariance requires uniform contribution of all frequencies. Then neither of them signals out any special line. Simple calculations show that this is possible if and only if $\alpha(f, T)$ is a linear function:

$$
\alpha(f, T)=1+k\left(f-\frac{1}{T}\right)
$$

where $k$ is a specific to a system parameter related to the properties of the U-turns.

If for example $\alpha(f, T)$ is a quadratic function, for every time window there is a frequency where the second derivative of the power spectrum shape changes its sign.

It should be stressed that the determination of the properties of the function $\alpha(f, T)$ does not involve any reference to nature of a system and to the statistics of the corresponding time series! Thus, the conclusion is that the shape $1 / f^{\alpha(f, T)}$ is independent from the statistics of the time series, from the nature of the system and from the length of the time window where the measurement is made. At the same time the linearity of the function $\alpha(f, T)$ ensures its time-translational invariance.

It should be stressed on the self-consistency of the above proof. Indeed, it starts with imposing the requirement about smoothness and continuity of the continuous band viewed as a necessary condition for avoidance of resonances. In turn, the boundedness alone turns sufficient to provide not only the smoothness of that shape and its universality, but the time translational invariance and long-term stability of the entire behavior of a complex system. A special attention must paid on the necessity that the condition about the uniform distribution of zeroes must hold on each and every scale so that to provide the smoothness of the shape of a continuous band and thus to all other derived from it properties. It is worth noting that this sequence of conclusions is an exclusive property of the concept of boundedness because the shape of the power spectrum of any unbounded sequence explicitly depends on the statistics of the corresponding time series.

Another far-going consequence of the condition about uniform distribution of zeroes is that it provides Euclideanity of the functional metrics by means of maintaining the countability of the number of zeroes and their uniformity.

At this point a question arises: the maintenance of permanent Euclideanity of the functional metrics implies that the local deviations from it must be sustained bounded and short-lived. However, this contradicts the fluctuation-dissipation theorem, according to which any response of any system exactly matches the development of an appropriate fluctuation. However, the latter implies that since the response is macroscopic, fluctuations also develop to macroscopic size. The 
way out is again highly non-trivial and passes through the utilization of a novel notion of chemical potential. The latter is necessary since simple considerations presented in [1] [2] [3] prove that the traditional notion of chemical potential is inherently contradictive. In turn, the novel notion of the chemical potential put forward by the author yields that all local fluctuations have bounded size and bounded lifetime [1] [2] [3]. In turn, the latter ensures that all internal fluctuations remain local and dissipate in microscopic time. In turn, their impact on macroscopic coherence turns out to be minor. Alongside, the latter ensures stability and robustness to the local details of the general physical protocol for self-sustaining the Euclideanity of the local functional metrics along with the execution of smooth U-turns on reaching the thresholds of stability. It should be stressed that the latter properties of the general physical protocol are exclusive for stationary homogeneous processes presented through stationary BIS only.

It is worth noting on the fundamental difference with the periodic functions utilized in the traditional approach for substantiation of the process of synchronization. Unlike the periodic functions which impose specific prerequisite structure on the entire space-time, i.e. an infinite set of nodes, stationary BIS, coarse-grained included, being irregular functions, impose coherence on bounded region of space-time only, that is the corresponding homeostatic pattern. At the same time the continuous band of a stationary BIS does not signals out any special component thus providing time-translational invariance of the functional relations encapsulated in the corresponding homeostatic pattern.

\subsection{Universal Coarse-Grained Distribution of a Stationary BIS}

Before focusing on the properties of the universal coarse-grained distribution of a stationary BIS let us consider the procedure for taking away that part of a BIS which belongs to its homeostatic pattern. To remind, while the power spectrum is additively decomposable, the homeostasis and the deviations from it are intertwined in the original BIS. Then, by means of making inverse Fourier transform of the discrete pattern, a periodic function which represents the homeostatic pattern alone is produced. By means of its extraction from the original BIS, a BIS which commences from the continuous part of the power spectrum is obtained. My assertion is that this part of a stationary BIS is subject to universal coarse-grained distribution.

Let us suggest that a coarse-grained structure exists. Since the boundedness of rates introduces a long-range persistence of the succession of jumps in the finer structure, the latter could be excellently approximated by fractal Brownian walks superimposed onto the coarse-grained structure. The major suggestion is that the universal coarse-grained structure consists of a train of excursions which forms a homogeneous stationary process and which acquire some exclusive properties set by the boundedness. Recalling that an excursion consists of a trajectory of walk originating at a given point at moment $t$ and returning to it for the first time at the moment $t+\Delta$. The characteristics of each excursion amplitude $A$, duration $\Delta$ and embedding interval $T$ are illustrated at Figure 1. 


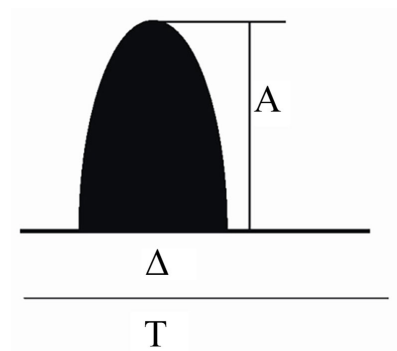

Figure 1. Characteristics of an excursion.

The embedding is a property which explicitly commences from the permanent smoothness of the shape of the continuous band of a stationary BIS. In a nutshell, the universality and the scale invariance of the coarse-grained distribution are derived on the exclusive grounds of the smoothness of the shape of the continuous band in the power spectrum which provides an exclusive shape of the autocorrelation function of a stationary BIS; details of its derivation can be found in Chapter 5 of [1]. Thus, the universality and the scale-invariance of a coarsegrained stationary BIS are immediately related to the condition about the uniform distribution of zeroes on each and every scale viewed as a condition for providing a long-term stable running of a complex system.

The notion of embedding implies that each excursion is embedded in a larger interval whose duration is interrelated with the duration of the excursion itself. The major role of the embedding is that it does not allow overlapping of the successive excursions and thus prevents growing of the excursion amplitude to arbitrary size. In turn, it results in permanent preservation of both boundedness of amplitude and boundedness of rates. Alongside, the disposition of an excursion onto its embedding interval is almost equi-probable along the entire interval. In turn, this prevents any periodicity in the sequences of excursions and thus provides scale invariance of BIS by means of making a coarse-grained BIS to be again a BIS.

It is worth noting that embedding is an exclusive property of a stationary BIS and has no analog for the unbounded sequences.

Then, the notion of an excursion along with the above consideration renders the size, the duration and the length of embedding interval of an excursion to be related by a power dependence of the following type:

$$
A \sim \Delta^{\alpha(\Delta)}
$$

The non-constant power $\alpha(\Delta)$ is set by the corresponding specific dynamics. Now the difference between a law and a general operational protocol becomes apparent. Indeed, a law viewed as quantified recursive relation among specific variables characterizing a given phenomenon put in a steady environment is expressed through the concrete values of the non-constant power $\alpha(\Delta)$ which thus quantifies the concrete relations between the size, the duration and the size of the embedding interval for any given excursion at current environment. Note that in an ever-changing environment the details of the 
non-constant power $\alpha(\Delta)$ could vary from one realization of the environment to another. On the other hand, the very existence of a universal scale-invariant coarse-grained structure of a stationary BIS, viewed as a part of the response of any stable complex system to an un-specified ever-changing environment is robust to the details of any concrete dynamics and the details of any concrete interaction with the environment. Thus, the above considerations confirm the difference between a law and an operational protocol with respect to their holding in a steady or in an ever-changing environment.

The idea that the coarse-grained structure of each and every BIS consists of embedded excursions which form a stationary homogeneous process implies that the frequency of occurrence of an excursion of size $A$ is time-independent and reads:

$$
P(A)=c A^{\alpha(A)} \frac{\exp \left(-A^{2} / \sigma^{2}\right)}{\sigma}
$$

The required probability $P(A)$ is given as a product of the term $A^{\alpha(A)}$ which is the statistical weight of an excursion of amplitude $A$ and its embedding interval, and the probability for appearance of excursion of that size (normal distribution); $\sigma$ is the variance of the BIS; $c=1 / \sigma^{\alpha(\sigma)}$ is the normalizing term. The homogeneity of the excursion succession ensures the time-independence of $P(A)$. It is worth noting that the Gaussian-like term $\frac{\exp \left(-A^{2} / \sigma^{2}\right)}{\sigma}$ in Equation (9) commences from the scale invariance of the coarse-grained structure of any BIS: a coarse-grained BIS is again BIS and this happens on each and every scale. Then, taking into account that the process is a stationary homogeneous one and fact that, according to the Lindeberg theorem [4] it has well-defined mean and variance, it turns out to be a subject to the normal distribution. It is worth noting that the latter does not imply that the succession of excursions consists of random independent events. On the contrary, the succession of excursions on any scale has residual memory which is dictated by the formation of larger excursions on the next scale. The residual memory is best pronounced through the appearance of some trend of the average on the development of an excursion. Yet, on elimination of a trend, excursions appear as random, independent events.

The universal distribution $P(A)$ from Equation (9) is presented in Figure 2. The dotted line represents the excellent approximation of the fat tail with power dependence. Yet, it is worth noting one of the greatest advantages of the universal distribution $P(A)$ from Equation (9) is that it is time-translational invariant unlike a power dependence which is in a conflict with it. More about this matter can be found in Chapter 5 of [1].

Let us now pay special attention to the self-consistency between obtained universal distribution of a stationary BIS and condition about the uniform distribution of the zeroes of a stationary BIS. Indeed, the universality of the coarse-grained distribution is derived on the exclusive grounds of the smoothness of the shape 


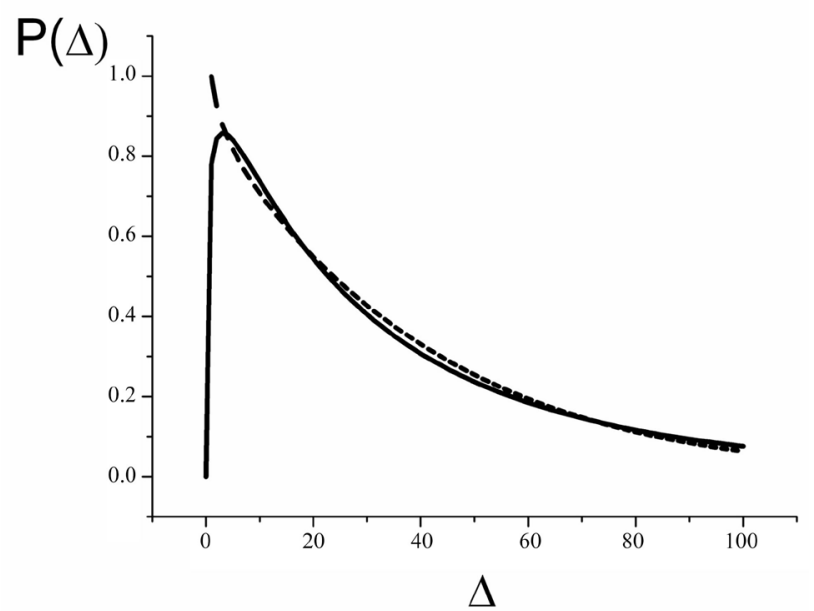

Figure 2. The shape of the universal distribution of a stationary BIS (continuous line) and approximation of its fat tail with a power dependence (dotted line).

of the continuous band in the power spectrum. Taking into account that the latter smoothness is an immediate consequence of imposing the condition about the uniform distribution of zeroes on each and every scale, the universal coarse-grained distribution is an immediate consequence of that condition. Vice versa: the constraint over the size and correspondingly duration of the largest excursions by the margins of stability and by the embedding render the number of zeroes in any window of recording of the corresponding BIS countable and independent from the length of that window which holds at each and every scale. Consequently, the latter provides a scale invariance of the uniform distribution of the zeroes of a BIS. In turn, the latter scale invariance results in the scale invariance of the universal distribution.

\section{Role of the Resolution of Measurements. Central Limit Theorem as Approximation}

At first glance the title of the present section sounds bizarre since the coarse-graining and the resolution of a measurement involve the same operations which consist of the notion that certain fine details become non-discernable. Yet, although that is correct, there is a fundamental difference between them: unlike the resolution which a solitary operation and thus not scale-invariant, the coarse-graining which yields the universal structure of a BIS is a scale-invariant operation. Next it is demonstrated that the superimposing of both operations results in mimicking the widest variety of behavior: from stochastic-like to periodic-like, to sequence of irregular fractals and to series of random independent events.

Thus, the stochastic-like behavior appears at very high resolution and relatively short time series. Then the role of the fine-structure becomes predominant. The distribution of the variations can be any one. Yet, most probably it is noisy and not well-defined. 
The behavior which mimics best a sequence of irregular fractals appears for intermediate resolutions both of the amplitude and time so that the shape and the durations of excursions to be recorded. Again, most probably the distribution of the excursions is not well-defined for relatively short time series.

A periodic-like behavior appears as a limit case of the fractal-like behavior. This happens at low resolution of the amplitudes of excursions so that almost only largest size excursions are discerned. Then, as a special occasion, the record could match an almost periodic train of impulses. The shape of the power spectrum is defined mainly by the shape of the corresponding impulse function.

Next in the line comes the case when the duration of the most of excursions is not discerned. Then, their appearance matches best random-like events so that a specific for each of them probability could be assigned. Yet, since the randomness is still approximation (remember the ubiquitous trend of local average), this probabilities could effectively vary in the course of recording. Thus, in the frame of the traditional theory of probability, such behavior would match rather a non-stationary process. However, the latter conclusion is in sharp contradiction with the reality since the underlying process is a stationary and homogeneous one. It is worth noting that a true non-stationary process is characterized by the appearance of an extra-line in a power spectrum [5]. Yet, the question whether it brings about adaptation or destruction is mathematically undecidable [5] and so it needs additional experiments for an irrefutable resolution.

This conflict is fundamental since it concerns the grounds of both the decomposition theorem and the Central Limit Theorem viewed as counterparts each of which establishes an asymptotic behavior for different and non-overlapping subjects. To remind, while the Central Limit Theorem holds for independent random variables (yet unbounded), the decomposition theorem holds for arbitrary variables provided the latter are bounded (yet not independent). The major clue for resolution of that conflict lies in the colocation "asymptotic behavior". Keeping in mind that the original times series is represented by a stationary BIS, the asymptotic behavior of the real process gradually approaches the asymptotic characteristics of a BIS, those are additively decomposed power spectrum and universal distribution of the members of the corresponding coarse-grained BIS. Practically, the later conclusion is verifiable by means of recording long enough time series. The guess is that when the length of a time series is much longer than the duration of the largest excursion, the asymptotic behavior of a BIS starts to reveal best. So, in this case the Central Limit Theorem appears as an approximation for relatively short time series while the asymptotic behavior is governed by the decomposition theorem for each and every of the above cases.

Outlining, the considerations in the present section are an excellent methodological example for the role of taking into account the grounds of any approach for the conclusions drawn from an experiment. Moreover, in order to make decisive conclusions, additional experiments are always necessary because the reading of any experiment strongly depends on the explanatory setting where it is put in and so it is not immunized from incorrect conclusions. 


\section{Conclusions}

A general operational protocol which provides permanent macroscopic coherence of the response of any stable complex system to an ever-changing environment is proposed. It turns out that the coherent response consists of two parts: 1) a specific discrete pattern, called by the author homeostatic one, whose characteristics are robust to the statistics of the environment; 2) the rest part of the response forms a stationary homogeneous process whose coarse-grained structure obeys universal distribution where the coarse-graining has the property to be scale-invariant.

The general physical protocol governing the dynamics of the spontaneously executed physico-chemical processes has been put forward by the author and consists in the following: local exceeds of energy are dissipated as emission of local acoustic phonons at a given spatio-temporal point and their absorption at another spatio-temporal point; local exceeds of matter are dissipated by means of targeted transportation of a specific constituents from a specific initial spatio-temporal point to a specific distant spatio-temporal point. Thus, the travel of matter waves between specific distant points serves as a general protocol for launching specific chemical reactions so that the input reagents and the output products are produced at distant points leaving at the same time the chemical reactions to be local events. Then, the global coherence appears as sequences of local emissions and absorptions of acoustic phonons and mater waves. The ubiquity of that mechanism is grounded on the ubiquity of the presence of the acoustic phonons and on the recently proved ubiquity of the matter wave emission in any system regardless to its nature and its physico-chemical characteristics.

Mathematically, the ubiquity of that protocol is established by the highly non-trivial properties of the new mathematical objects called stationary BIS (bounded irregular sequences) introduced for its description. The major of those properties consists of the fact that the coarse-grained structure of a stationary BIS is scale invariant and robust to the nature of system, its dynamics and the details of the environmental impact. The latter implies that the fine structure of a stationary BIS has minor effect on the coarse-grained one. In turn, the latter substantiates the ubiquitous availability of the proposed general protocol of coherence to systems of different nature, different dynamics etc. It is worth noting that this property of BIS is exclusive for them and it is not shared by periodic functions. Moreover, the BIS remain stable under local perturbations unlike their periodic counterparts where even tiniest local perturbations of the characteristics of a periodic function affect the entire function. The latter implies that local perturbations have global impact on any periodic function on the contrary to the stationary BIS where a local perturbation remains local and have minor effect on the coarse-grained structure.

Moreover, the universality of the coarse-grained structure provides uniform asymptotic behavior for systems of different nature and dynamics which is go- 
verned by the recently proven by the author decomposition theorem.

The high non-triviality of that mater consists of the consideration that for relatively short time series, a measurement, viewed as a solitary operation of coarse-graining, superimposed on the universal distribution results in a rich variety of behaviors ranging from a periodic-like to a stochastic-like, to sequences of irregular fractal-like objects and sequences of random-like events. The relevance of Central Limit theorem applies to the latter case. This, to certain extent unexpected, rich variety of behaviors is a direct consequence of the superimposing of the resolution, viewed as a solitary scale-non-invariant operation of coarse-graining, and the scale-invariance of coarse-graining which yields the universal distribution. Then, the application of the Central Limit Theorem is relevant only as an approximation which holds for relatively short time series and specific low resolution of the measurement equipment while the asymptotic behavior in each and every of the above cases is governed by the decomposition theorem.

Outlining, the present paper appears as an excellent methodological example for the role of taking into account the grounds of any approach for the conclusions drawn from an experiment. Moreover, in order to make decisive conclusions, additional experiments are always necessary because the reading of any experiment strongly depends on the explanatory setting where it is put in and so it is not immunized from misleading conclusions.

\section{Conflicts of Interest}

The author declares no conflicts of interest regarding the publication of this paper.

\section{References}

[1] Koleva, M.K. (2012) Boundedeness and Self-Organized Semantics: Theory and Applications, IGI-Global, Hershey, PA. https://doi.org/10.4018/978-1-4666-2202-9

[2] Koleva, M.K. (2021) Journal of Modern Physics, 12, 167-178. https://doi.org/10.4236/jmp.2021.123015

[3] Koleva, M.K. (2005) Fluctuations and Long Term Stability: From Coherence to Chaos. https://arxiv.org/abs/physics/0512078v1

[4] Feller, W. (1970) An Introduction to Probability Theory and Its Applications. John Willey \& Sons, New-York.

[5] Koleva, M.K. (2020) Journal of Modern Physics, 11, 767-778. https://doi.org/10.4236/jmp.2020.116049 